\title{
Secondary lymphoblastic leukemia occurring 38 months after the primary diagnosis of multiple myeloma: A case report
}

\author{
JUNXUN LI $^{1}$, JIEYU ZHAN ${ }^{2}$, FAN ZHANG ${ }^{1}$, ZHUANGJIAN YE ${ }^{1}$ and JUAN OUYANG ${ }^{1}$ \\ ${ }^{1}$ Department of Laboratory Science, The First Affiliated Hospital of Sun Yat-sen University, Guangzhou, Guangdong 510080; \\ ${ }^{2}$ Department of Pediatrics, The First Hospital of Baiyun, Guangzhou, Guangdong 510410, P.R. China
}

Received November 9, 2014; Accepted May 5, 2016

DOI: $10.3892 / 01.2016 .4728$

\begin{abstract}
Multiple myeloma (MM) is a malignant plasma cell neoplasm characterized by the accumulation of plasma cells in the bone marrow, the subsequent destruction of bone and organ dysfunction. The present study describes the case of a 66-year-old male patient who presented with the typical clinical manifestations of MM. The patient was administered a bortezomib and dexamethasone regimen for 2 cycles and achieved complete remission. Lenalidomide, vincristine, pirarubicin, dexamethasone, melphalan and thalidomide was used successively in consolidation therapy and maintenance therapy. The patient developed secondary B-cell lymphoblastic leukemia 38 months after the primary MM diagnosis was made. Owing to the exposure of the patient to a variety of therapeutic agents, it could be inferred that multiple immune defects may have played an important role in the secondary lymphoblastic leukemia of the patient. Microscopic examination and flow cytometry detection were important in identifying the secondary malignancy in this MM case.
\end{abstract}

\section{Introduction}

Multiple myeloma (MM) is a malignant plasma cell neoplasm characterized by plasma cells accumulating in the bone marrow and subsequent destruction of bone, symptoms

Correspondence to: Dr Junxun Li, Department of Laboratory Science, The First Affiliated Hospital of Sun Yat-sen University, 58 Zhongshan Er Road, Guangzhou, Guangdong 510080, P.R. China E-mail: abericcc@126.com

Dr Jieyu Zhan, Department of Pediatrics, The First Hospital of Baiyun, 1128 Jichang Road, Guangzhou, Guangdong 510410, P.R. China

E-mail: sanagirl@126.com

Abbreviations: MM, multiple myeloma; MBS, metastatic bone survey; $\mathrm{CD}$, cluster of differentiation; AML, acute myeloid leukemia; MDS, myelodysplastic syndromes

Key words: multiple myeloma, secondary malignancy, B-cell lymphoblastic leukemia of bone marrow failure and organ dysfunction (1). MM is responsible for $\sim 1 \%$ of all cancers and $10 \%$ of hematological cancers $(2,3)$. The median age at diagnosis of $\mathrm{MM}$ is 70 years (3). The median survival of patients with MM was $<1$ year prior to the introduction of alkylating agents (3). The introduction of novel agents, including bortezomib, thalidomide and lenalidomide, for the treatment of MM patients has significantly improved clinical outcomes (1-3). However, a concerning finding has been the increase in the incidence of secondary malignancies (4-7). Previous studies have demonstrated that MM patients have a higher risk of secondary myeloid malignancies than the general population (4-6). To the best of our knowledge, secondary B cell malignancy in MM patients is seldom studied. In addition, acute lymphoblastic leukemia (ALL) is often perceived as a pediatric malignancy because the peak incidence occurs between 1 and 4 years of age. However, the incidence of ALL has increased in the older population (8).

The present authors previously reported 3 cases of MM who developed lymphoblastic leukemia after exposure to a variety of agents (9). The present study reports the case of a patient who developed B-cell lymphoblastic leukemia 38 months after the initial diagnosis of MM. A brief review of the literature is also provided. Written informed consent was obtained from the patient for the publication of this case report and any accompanying images.

\section{Case report}

Clinical characteristics. A 66-year-old male was admitted to The First Affiliated Hospital of Sun Yat-sen University (Guangzhou, China) in June 2010 due to bone pain. A metastatic bone survey (MBS) revealed focal osteolytic bone lesions in the costal bones, spine, pelvis, clavicle and scapular of the right side (Fig. 1). Complete blood counts showed normal results for white blood cells $\left(4.68 \times 10^{9}\right.$ cells $/ 1$; normal range, $4.00-10.00 \times 10^{9}$ cells/l) hemoglobin (130 g/l; normal range, $120-160 \mathrm{~g} / \mathrm{l})$ and platelet count $\left(172 \times 10^{9}\right.$ cells/1; normal range, $100-300 \times 10^{9}$ cells/1). A blood smear showed a rouleaux formation of red blood cells. Serum protein electrophoresis revealed the presence of a monoclonal protein in the $\gamma$ region, subsequently identified as immunoglobulin (Ig) A and $\kappa$ light chain. A total of $16.00 \%$ plasma cells (normal range, 0.00 $0.02 \%$ ) were found in the bone marrow smear (Fig. 2). 


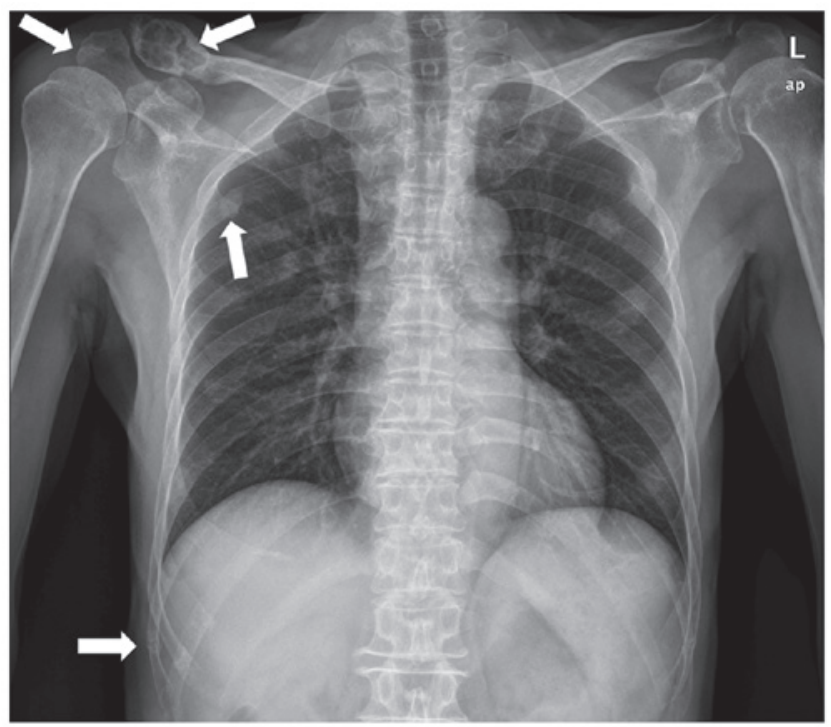

Figure 1. Focal osteolytic bone lesions in costal bones, the clavicle and the scapular acromion process (white arrows).

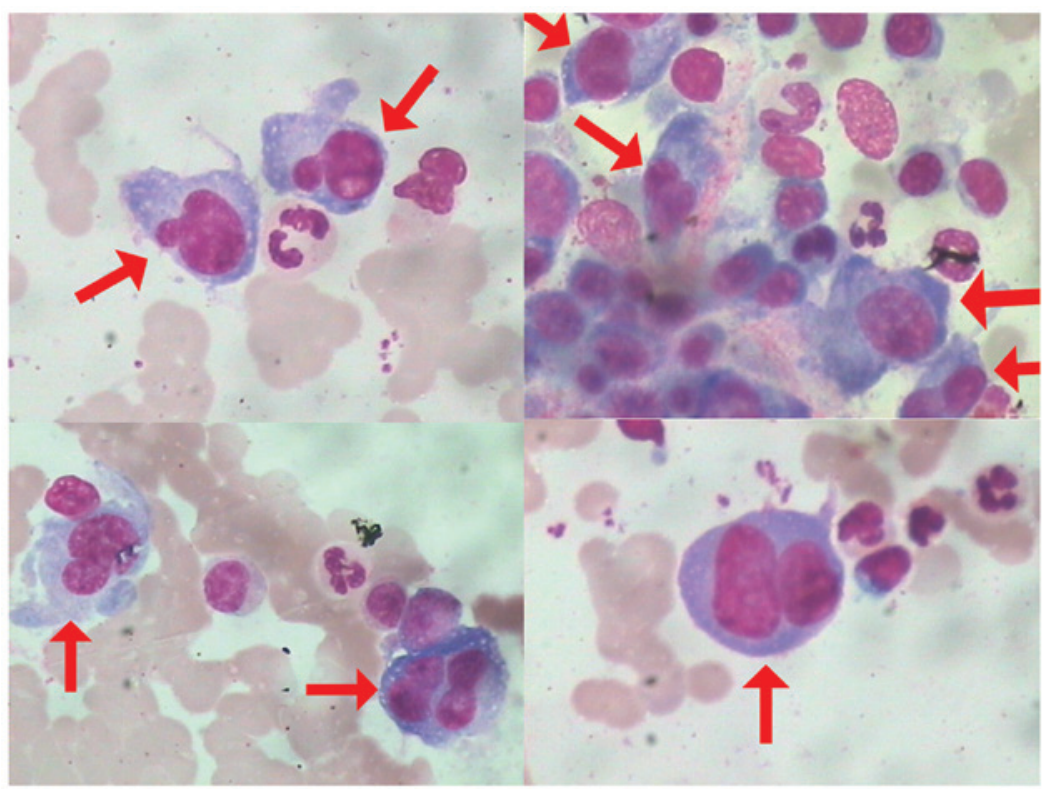

Figure 2. Plasma cell morphology in the bone marrow smear at diagnosis (Wright-Giemsa staining; magnification, x1,000). Abnormal plasma cells (red arrows) account for $16 \%$ of the nucleated cells in the bone marrow. The abnormal plasma cells are characterized by large and irregular nuclei, fine chromatin, occasional nucleoli and atypical cytoplasmic features.

The results of flow cytometry analysis were consistent with the presence of clonal plasma cells. Flow cytometry analysis of the surface and cytoplasm markers of the plasma cells was performed on erythrocytes-lysed ethylenediaminetetraacetic acid (EDTA)-anti-coagulated bone marrow samples using a 6-color panel of ready to use antibodies and isotype controls conjugated to fluorescein isothiocyanate (FITC), phycoerythrin (PE), peridinin chlorophyll (PerCP), PE-cyanine 7 (Cy7), allophycocyanin (APC) and/or APC-Cy7 (BD Biosciences, San Diego, CA, USA) and the "Duo-Lyse" program of the FACS ${ }^{\mathrm{TM}}$ Lyse Wash Assistant (BD Biosciences, Franklin Lakes, NJ, USA) according to the following antibody combinations: For plasma cells analysis, i) anti-CD38/ CD56/CD19/CD20/CD138/CD45; ii) anti-cytoplasmic (c)К/ c $\lambda / C D 19 / C D 138 / C D 38 / C D 45 ;$ iii) anti-CD38/CD54/CD138/ CD45; and iv) anti-CD38/CD45/CD56/CD19/CD20 were used, while for lymphocyte analysis, i) anti-CD10/CD34/human leukocyte antigen-antigen D related/CD45; ii) anti-CD20/ CD22/CD45/CD5/CD19; iii) anti-CD15/CD117/CD14/CD45/ CD19; iv) anti-CD56/CD13/CD33/CD45/CD19; v) anti-cCD3/ myeloperoxidase (MPO)/CD79a/CD45; and vi) anti-CD2/ CD7/CD45/CD19/CD3 were used (Fig. 3 and Table I). The total Bence-Jones protein level in a 24-h urine collection was $1,740 \mathrm{mg}$ (reference value, negative). Anti-nuclear antibody and serological tests (Epstein-Barr, hepatitis B and C viruses, cytomegalovirus and human immunodeficiency virus) were negative. As a result of all these findings, the patient was diagnosed with MM (Durie-Salmon IIA) (10). 
Table I. Antibodies used for flow cytometry.

\begin{tabular}{|c|c|c|}
\hline Antibodies & Catalogue number & Concentration $(\mu 1 / 100 \mu 1)$ \\
\hline Anti-CD38-FITC/CD56-PE-Cy/CD19-PerCP-Cy5.5 & 341132 & 20 \\
\hline Anti-CD20-PE-Cy7 & 335793 & 5 \\
\hline Anti-CD38 (HB-7)-APC & 345807 & 5 \\
\hline Anti-CD54-PE & 555511 & 20 \\
\hline Anti-CD138-PerCP-Cy5.5 & 341087 & 20 \\
\hline Anti-к-FITC/ $\lambda$-PE & 349516 & 20 \\
\hline Anti-IgM-FITC & 555782 & 20 \\
\hline Anti-IgG-FITC & 555786 & 20 \\
\hline Anti-CD19 (SJ25C1)-APC & 340437 & 5 \\
\hline Anti-CD22-PE & 347577 & 20 \\
\hline Anti-CD34 (8G12)-PE & 348057 & 20 \\
\hline Anti-CD10 (HI10a)-FITC & 340925 & 20 \\
\hline Anti-CD20-FITC & 347673 & 20 \\
\hline Anti-CD45-APC & 340943 & 5 \\
\hline Anti-CD45 (2D1)-PerCP-Cy5.5 & 347464 & 20 \\
\hline Anti-HLA-DR-APC & 559866 & 20 \\
\hline Anti-CD3-FITC/MPO-PE/CD79a-PerCP-Cy5.5 & 340961 & 20 \\
\hline Anti-CD20-FITC & 555622 & 20 \\
\hline Anti-CD2 (S5.2)-FITC & 347593 & 20 \\
\hline Anti-CD3-PE-Cy7 & 341091 & 5 \\
\hline Anti-CD5-APC & 340583 & 5 \\
\hline Anti-CD7-PE & 340581 & 20 \\
\hline Anti-MPO-FITC & 340580 & 20 \\
\hline Anti-CD13-PE & 347837 & 20 \\
\hline Anti-CD33-APC & 551378 & 20 \\
\hline Anti-CD117-PE & 555714 & 5 \\
\hline Anti-CD117 (104D2)-PerCP-Cy5.5 & 333944 & 20 \\
\hline Anti-CD56-FITC & 340410 & 20 \\
\hline Anti-CD15-FITC & 555401 & 20 \\
\hline
\end{tabular}

$\mathrm{CD}$, cluster of differentiation; FITC, fluorescein isothiocyanate; PE, phycoerythrin; PerCP, peridinin chlorophyll; Cy, cyanine; APC, allophycocyanin; Ig, immunoglobulin; MPO, myeloperoxidase; HLA-DR, human leukocyte antigen-antigen D related.

Treatment process. Treatment was commenced in June 22, 2010. At first, the patient was administered a bortezomib and dexamethasone regimen for 2 cycles (cycle 1: $2.0 \mathrm{mg}$ bortezomib on days 1, 4, 7 and 11, and $20 \mathrm{mg}$ /day dexamethasone on days $1,2,4,5,7,8,11$ and 12 , every 28 days; and cycle 2 : $2.0 \mathrm{mg}$ bortezomib on days $1,8,15$ and 22 , and $20 \mathrm{mg}$ /day dexamethasone on days $1,2,8,9,15,16,22$ and 23, every 28 days). The patient achieved complete remission (Figs. 4 and 5). Subsequent to obtaining written informed consent, lenalidomide (10 mg/day) was administered as maintenance therapy on days 1-21 every 28 days since September 15, 2010. However, due to economic reasons, lenalidomide was stopped 2 months later according to the patient's wishes. Therefore, a vincristine, pirarubicin, dexamethasone and melphalan regimen $(0.5 \mathrm{mg} /$ day vincristine, $10 \mathrm{mg}$ /day pirarubicin, $20 \mathrm{mg} /$ day dexamethasone and $12 \mathrm{mg} /$ day melphalan, on days 1-4) was administered every 28 days as consolidation therapy in November 16, 2010. After 1 cycle, the melphalan was out of stock, and a vincristine, pirarubicin and dexamethasone regimen $(0.5 \mathrm{mg}$ vincristine,
$10 \mathrm{mg}$ pirarubicin and $20 \mathrm{mg}$ dexamethasone, on days 1-4, every 28 days) was administered in December 27, 2010, for 1 cycle instead. Thalidomide ( $200 \mathrm{mg} /$ day) was used as maintenance therapy from February 9, 2011, to September 13, 2013, when the patient was admitted to hospital again due to serious fatigue that had persisted for $>10$ days.

Secondary lymphoblastic leukemia. At this time, the patient's blood counts showed a low white blood cell count of $3.05 \times 10^{9} / 1$, while the hemoglobin level $(140 \mathrm{~g} / \mathrm{l})$ and platelet count $\left(178 \times 10^{9} / 1\right)$ were normal. The serum protein level was $74.8 \mathrm{~g} / 1$ (normal range 60.0-80.0 g/l; albumin, $44.8 \mathrm{~g} / \mathrm{l}$ (normal range, 40.0-55.0 g/l); and globulin, $30 \mathrm{~g} / \mathrm{l}$ (normal range, $20-30 \mathrm{~g} / \mathrm{l}$ ). Immunoelectrophoresis showed no monoclonal increase in serum $\operatorname{Ig}(\operatorname{Ig} \mathrm{A}, 1.52 \mathrm{~g} / \mathrm{l})$. The patient exhibited elevated levels of $\beta 2$-microglobulin $(2,358.1 \mu \mathrm{g} / \mathrm{l}$; normal range, $0-2,400 \mu \mathrm{g} / \mathrm{l})$, liver enzymes [alanine transaminase, $64 \mathrm{U} / 1$ (normal range, $0-40 \mathrm{U} / 1$ ); and aspartate transaminase, $76 \mathrm{U} / 1$ (normal range, $0-40 \mathrm{U} / \mathrm{l})]$ and creatinine $(77 \mu \mathrm{mol} / \mathrm{l}$; normal range, 

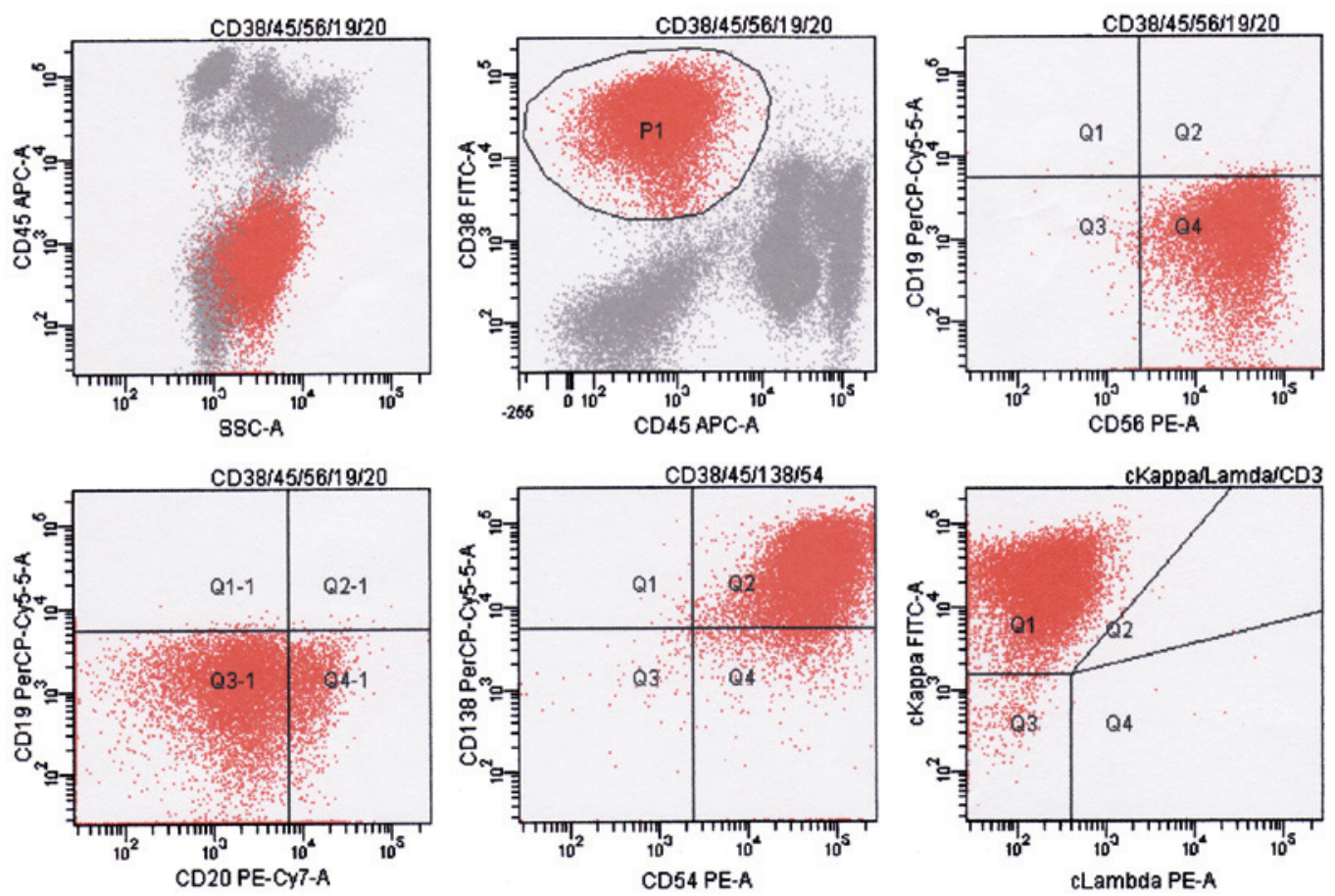

Figure 3. Result of flow cytometry analysis at diagnosis. P1 indicates abnormal plasma cells; the phenotype was positive for CD38, CD138, CD56, CD54 and $\mathrm{c} \kappa$ light chain, and negative for CD45, CD19 and CD20. Flow cytometry analysis of the plasma cells surface and cytoplasm markers was performed on erythrocytes-lysed ethylenediaminetetraacetic acid-anti-coagulated bone marrow samples using the "Duo-Lyse" program of the BC FACS" Lyse Wash

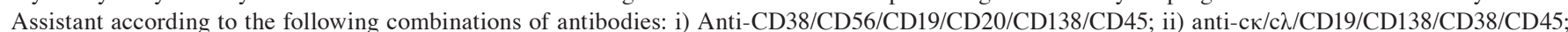
iii) anti-CD38/CD54/CD138/CD45; and iv) anti-CD38/CD45/CD56/CD19/CD20. The data were analyzed with BD FACSCanto ${ }^{\mathrm{TM}}$. The axes of the graphs represent fluorescence intensity data collected in pulse-area measurements and plotted on a logarithmic scale. CD, cluster of differentiation; c, cytoplasmic; A, area; SSC, side scatter; APC, allophycocyanin; PE, phycoerythrin; Cy, cyanine; PerCP, peridinin chlorophyll; FITC, fluorescein isothiocyanate; Q, quadrant.

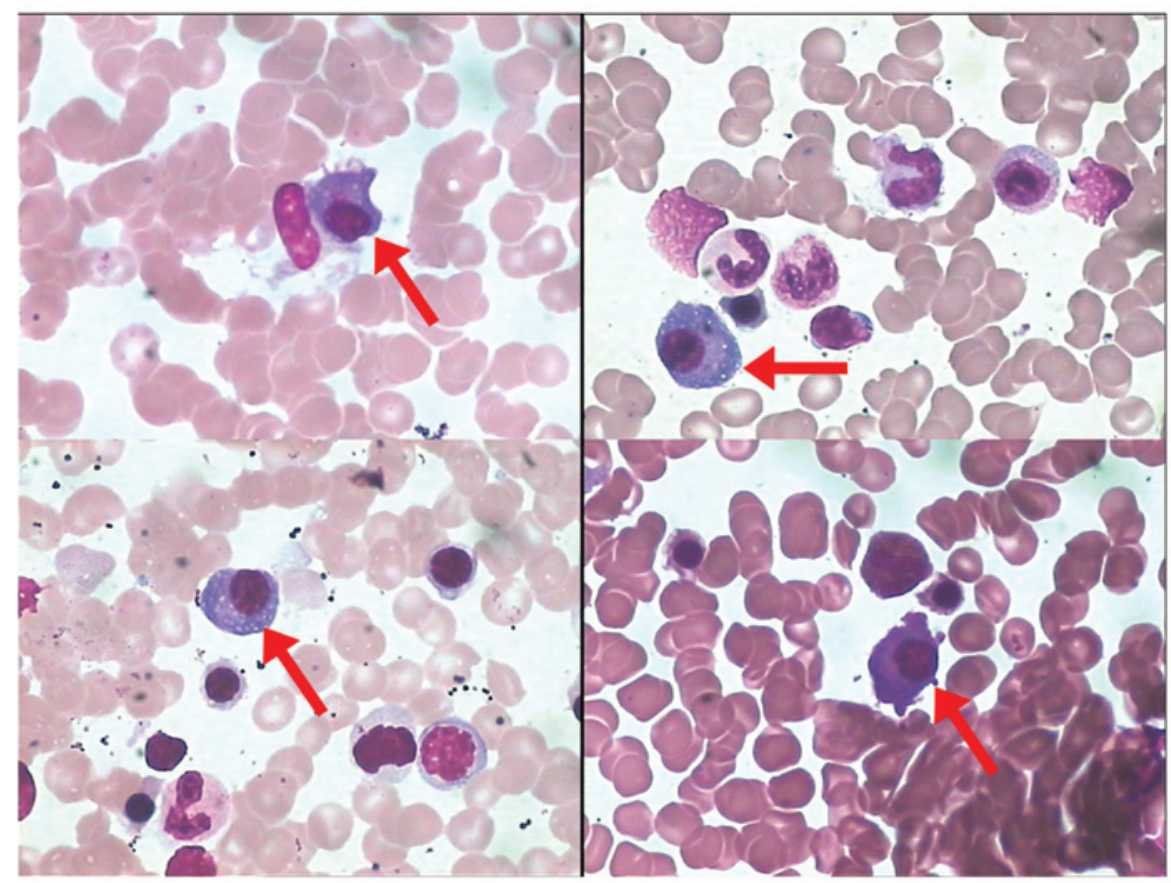

Figure 4. Plasma cell morphology in bone marrow smears after treatment (Wright-Giemsa staining; magnification, $\mathrm{x} 1,000$ ). The plasma cells accounted for $1 \%$ of nucleated cells. The cells are characterized by small nuclei, clumped chromatin, non-existent nucleoli and abundant cytoplasm (red arrows).

53-106 $\mu \mathrm{mol} / \mathrm{l})$, and a normal calcium level $(2.31 \mathrm{mmol} / \mathrm{l}$; normal range, $2.25-2.75 \mathrm{mmol} / \mathrm{l}$ ).

Lymphoblasts accounted for $62 \%$ of nucleated cells in the patient's bone marrow smear (Fig. 6). The results of flow cytometry analysis [positive for CD45, CD19, CD22, CD34 and $\mathrm{CD} 79 \mathrm{a}( \pm)$; and negative for CD38, CD138, CD56 and CD54] were consistent with the presence of clonal lymphoblast (Fig. 7). Flow cytometry analysis of the lymphoblast 

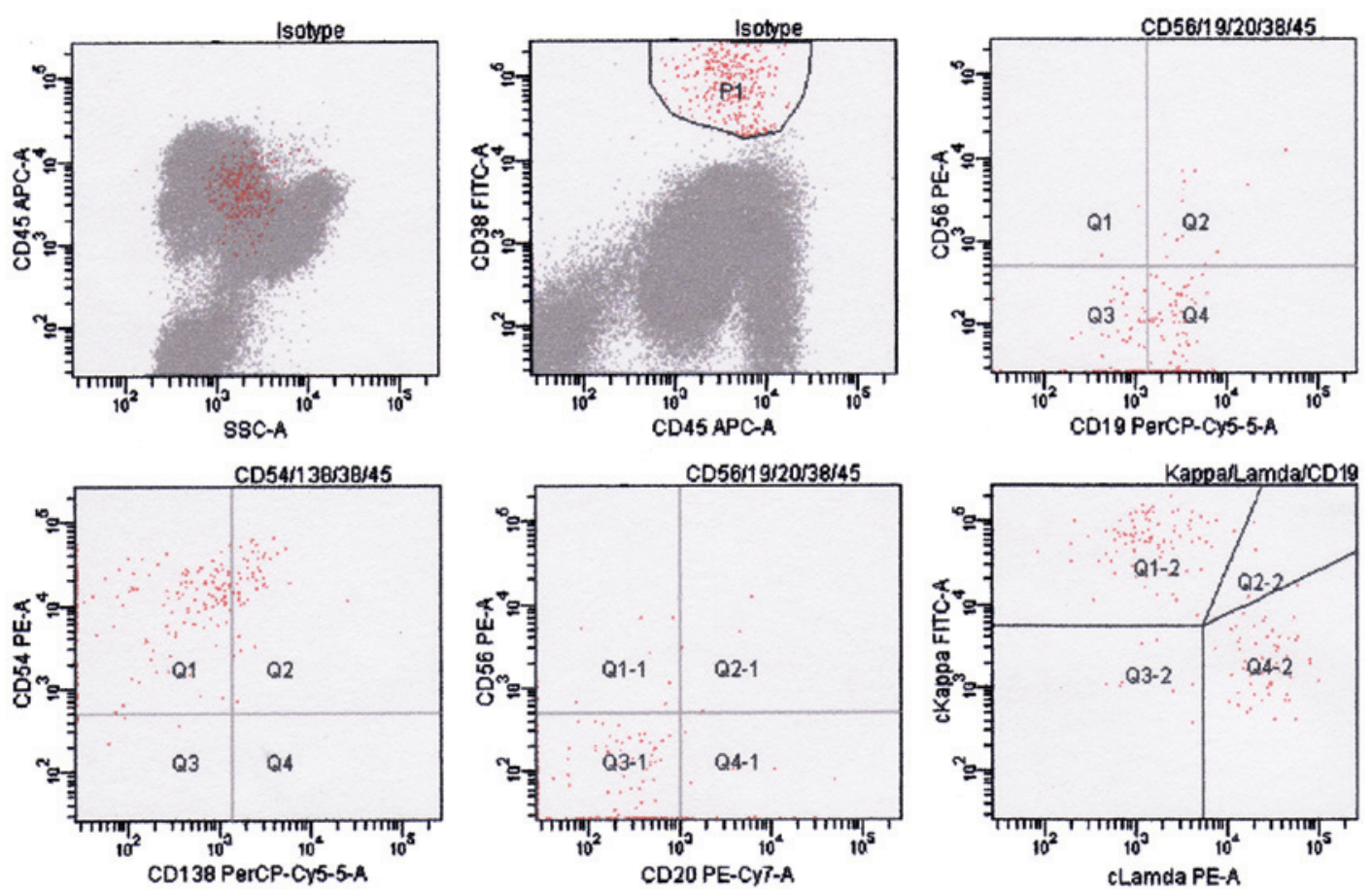

Figure 5. Result of flow cytometry analysis after treatment. P1 indicates plasma cells; the phenotype was positive for CD38, CD138, CD45, CD19 and CD54, and negative for CD56 and CD20. Flow cytometry analysis of the plasma cells surface and cytoplasm markers was performed on erythrocytes-lysed ethylenediaminetetraacetic acid-anti-coagulated bone marrow samples using the "Duo-Lyse" program of the BC FACS ${ }^{\mathrm{TM}}$ Lyse Wash Assistant according to the

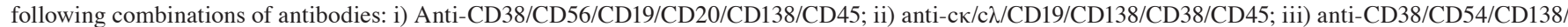
CD45; and iv) anti-CD38/CD45/CD56/CD19/CD20. The data were analyzed with BD FACSCanto ${ }^{\mathrm{TM}}$. The axes of the graphs represent fluorescence intensity data collected in pulse-area measurements and plotted on a logarithmic scale. CD, cluster of differentiation; c, cytoplasmic; A, area; SSC, side scatter; APC, allophycocyanin; PE, phycoerythrin; Cy, cyanine; PerCP, peridinin chlorophyll; FITC, fluorescein isothiocyanate; Q, quadrant.

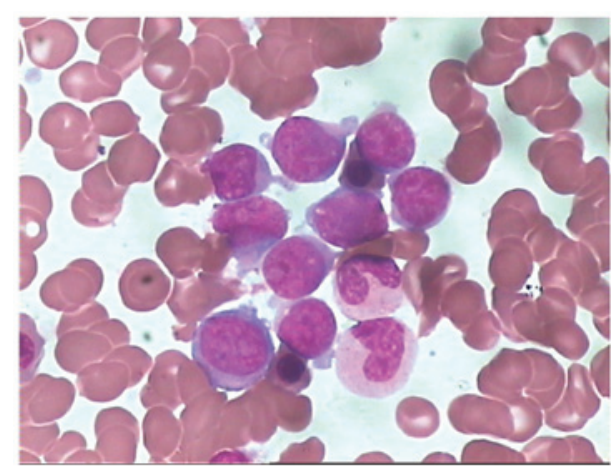

Figure 6. Lymphoblast morphology in a bone marrow smear 38 months after the multiple myeloma diagnosis (Wright-Giemsa staining; magnification, $\mathrm{x} 1,000)$. Lymphoblasts account for $62 \%$ of the nucleated cells, characterized by a consistent size, regular nuclei, small nucleoli and a small amount of cytoplasm.

surface and cytoplasm markers was performed on erythrocytes-lysed EDTA-anti-coagulated bone marrow samples using a 6-color panel of antibodies (FITC/PE/PerCP/PE-Cy7/ APC/APC-Cy7) and the "Duo-Lyse" program of the of the FACS ${ }^{\mathrm{TM}}$ Lyse Wash Assistant according to the following combinations of antibodies: i) Anti-CD10/CD34/CD19/CD20/ CD22/CD45 and ii) anti-cIgM/CD79a/CD34/CD19/CD45. For each antibody, negative staining levels were set by comparison with an isotype matched control. A cutoff of $20 \%$ was usually accepted as evidence of antigen expression (+). A positive percentage between 10 and $20 \%$ was usually accepted as evidence of antigen expression $( \pm)$.
Biopsy revealed a diffuse growth pattern of lymphoblasts in the bone marrow, and immunohistochemistry showed the following results: Terminal deoxynucleotidyl transferase(+), CD34(+), CD79a(土), CD3(-), CD5(-), MPO(-), CD56(-), CD138(-), cyclin D1(-), $\kappa(-)$ and $\lambda(-)$ (Fig. 8). MBS showed that focal bone lesions remained, and positron emission tomography-computed tomography revealed an elevated maximum standardized uptake value of fluorodeoxyglucose in the axial bone (including the skull and the vertebral column; maximum standardized uptake value, 3.6) and the spleen (maximum standardized uptake value, 2.6) (Fig. 9). The patient was negative for the BCR/ABL and IGH/ CCND1 genes on fluorescence in situ hybridization (FISH) tests. However, on karyotype analysis, a fragment from an unknown source that was an addition to chromosome 4 was observed in 2 out of the 7 analyzed cells (Fig. 10). Bone marrow metaphase cytogenetic studies were performed on 24-h bone marrow cultures without any colony stimulating factor. The cells were cultured in RPMI-1640 medium (Invitrogen; Thermo Fisher Scientific, Inc., Waltham, MA, USA) supplemented with $20 \%$ fetal calf serum (BD Biosciences) and $2 \%$ L-glutamine. The cells were harvested, and cell suspensions were stored in a freezer at $\sim-20^{\circ} \mathrm{C}$. Conventional cytogenetic karyotyping was performed using standard G-banding cytogenetic methods. Seven metaphases were analysed. FISH procedures were carried out on fixed bone marrow cells according to the manufacturer's protocol (Vysis; Abbott Molecular, Des Plaines, IL, USA). The slide was washed in $2 \mathrm{X}$ saline sodium citrate for $4 \mathrm{~min}$, followed 

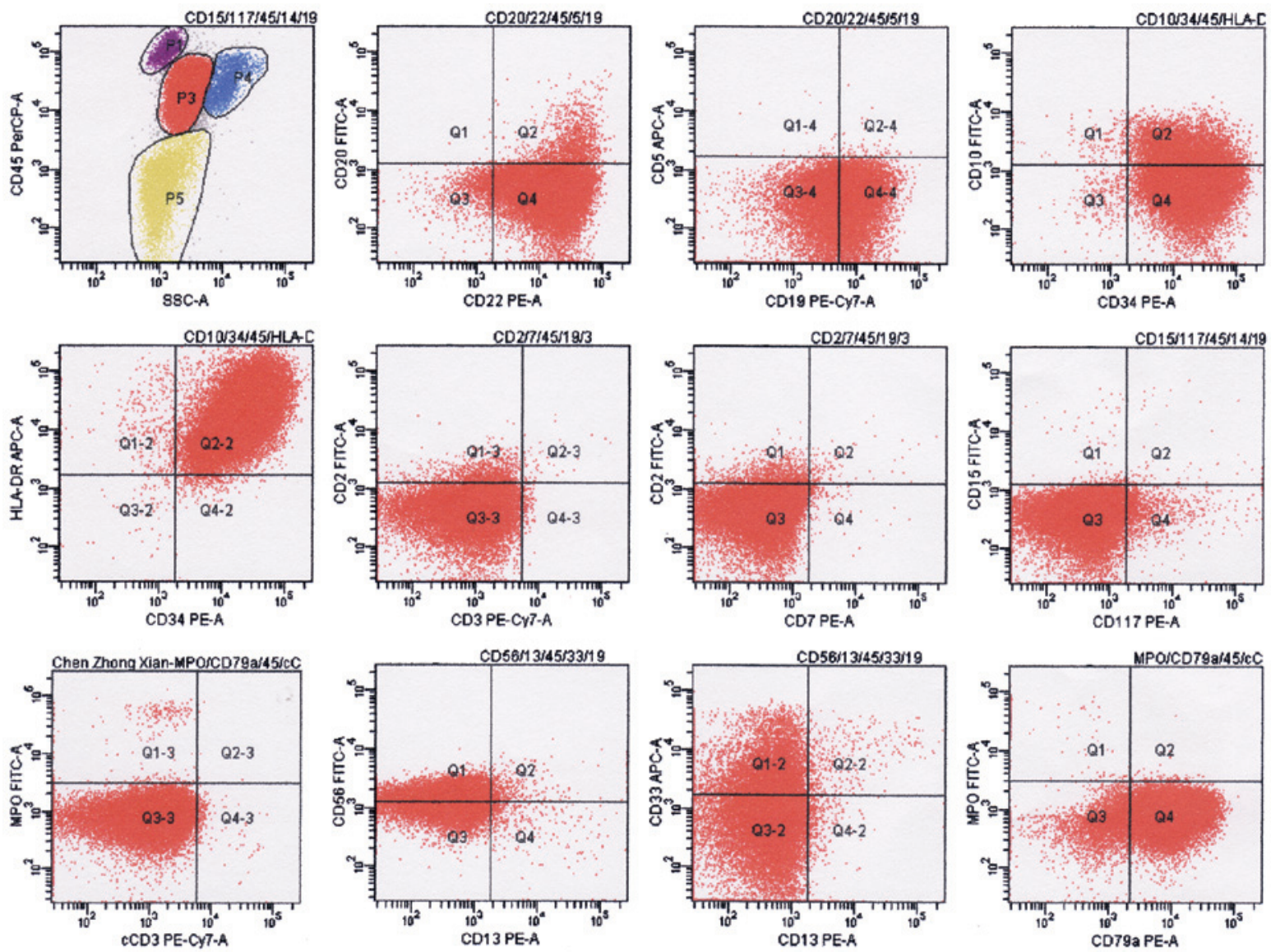

Figure 7. Result of flow cytometry analysis at the diagnosis of secondary malignancy. P3 indicate lymphoblasts; the phenotype was positive for CD19, CD22, CD79a, CD34, HLA-DR, CD56, CD10( \pm ) and CD33( \pm ); negative for MPO, cCD3, CD20, CD5, CD2, CD3, CD7, CD117, CD15 and CD13. Flow cytometry analysis of the plasma cells surface and cytoplasm markers was performed on erythrocytes-lysed ethylenediaminetetraacetic acid-anti-coagulated bone marrow samples using the "Duo-Lyse" program of the BC FACS ${ }^{\mathrm{TM}}$ Lyse Wash Assistant according to the following combinations of antibodies: i) Anti-CD10/CD34/ HLA-DR/CD45; ii) anti-CD20/CD22/CD45/CD5/CD19; iii) anti-CD15/CD117/CD14/CD45/CD19; iv) anti-CD56/CD13/CD33/CD45/CD19; v) anti-cCD3/ $\mathrm{MPO} / \mathrm{CD} 79 \mathrm{a} / \mathrm{CD} 45$; and vi) anti-CD2/CD7/CD45/CD19/CD3. The data were analyzed with BD FACSCanto ${ }^{\mathrm{TM}}$. The axes of the graphs represent fluorescence intensity data collected in pulse-area measurements and plotted on a logarithmic scale. CD, cluster of differentiation; c, cytoplasmic; A, area; SSC, side scatter; APC, allophycocyanin; PE, phycoerythrin; Cy, cyanine; PerCP, peridinin chlorophyll; FITC, fluorescein isothiocyanate; MPO, myeloperoxidase; HLA-DR, human leukocyte antigen-antigen D related; Q, quadrant.
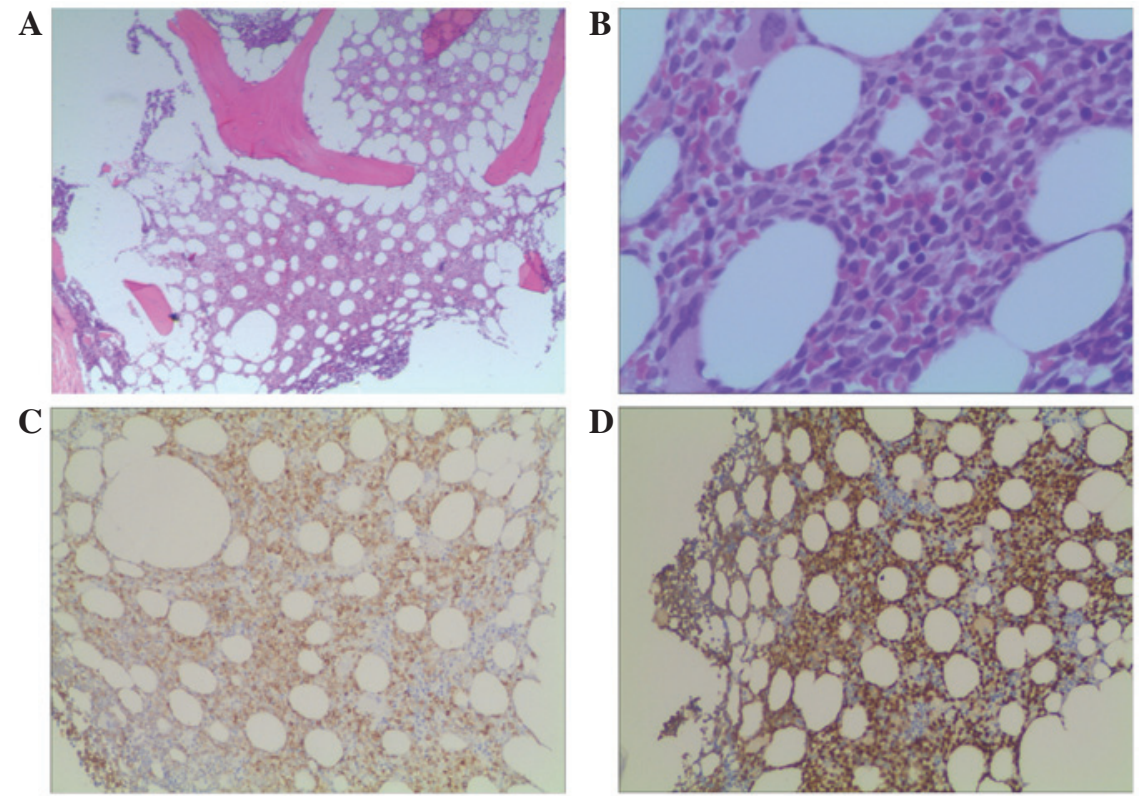

Figure 8. Microscopic appearance of the bone marrow biopsy. (A and B) A diffuse growth pattern of lymphoblasts in the bone marrow, characterized by a consistent size, fine chromatin and small nucleoli [hematoxylin and eosin staining; magnification, (A) x40 and (B) x400]. Immunohistochemical analysis results showing $(\mathrm{C})$ cluster of differentiation $79 \mathrm{a}( \pm)$ (magnification, $\mathrm{x} 40)$ and $(\mathrm{D})$ terminal deoxynucleotidyl transferase $(+)$ (magnification, $\mathrm{x} 40$ ). 


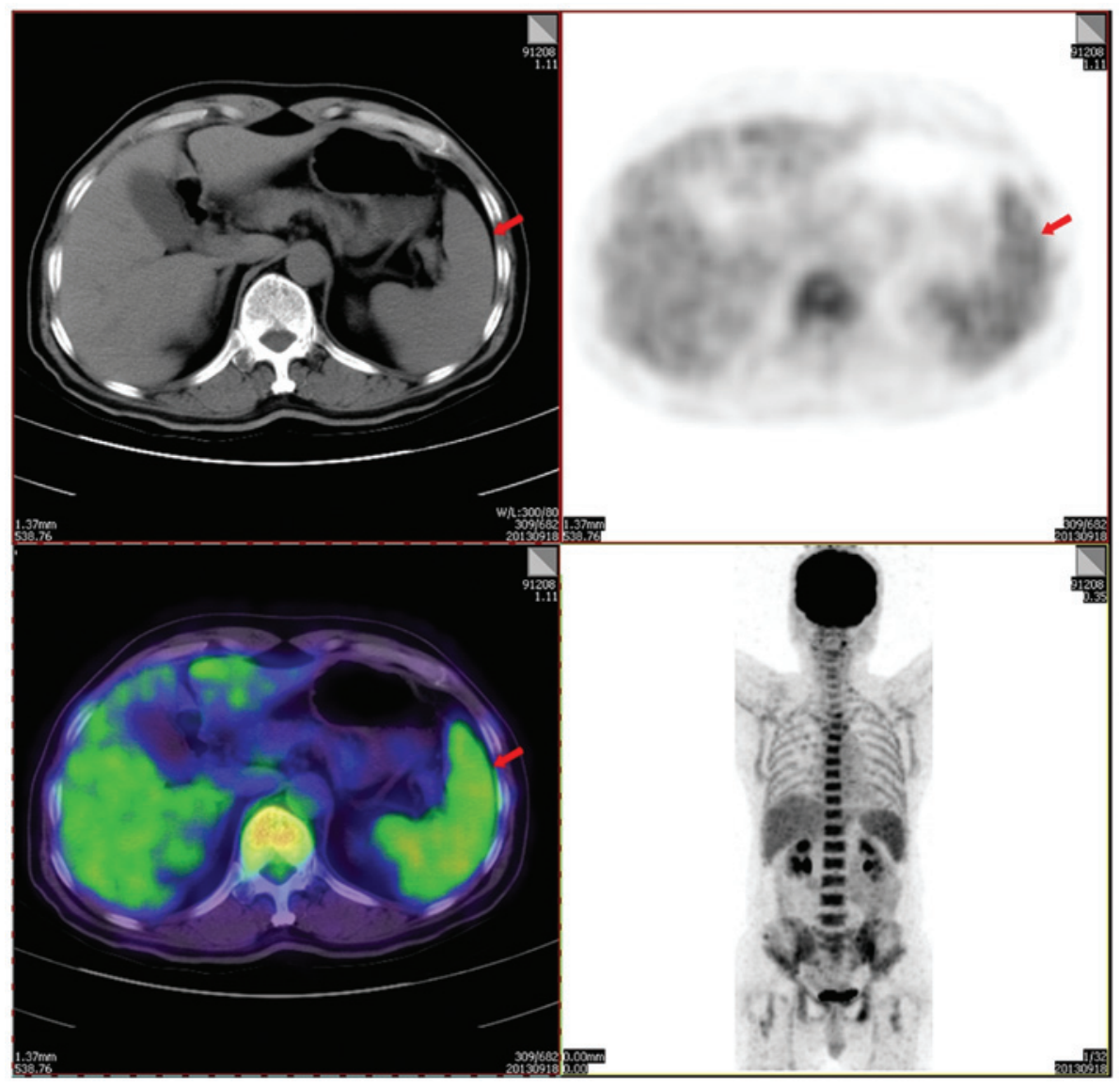

Figure 9. Positron emission tomography-computed tomography showing elevated maximum standardized uptake values of fluorodeoxyglucose in the axial bone (3.6) and spleen (2.6, as indicated by the red arrow).

A
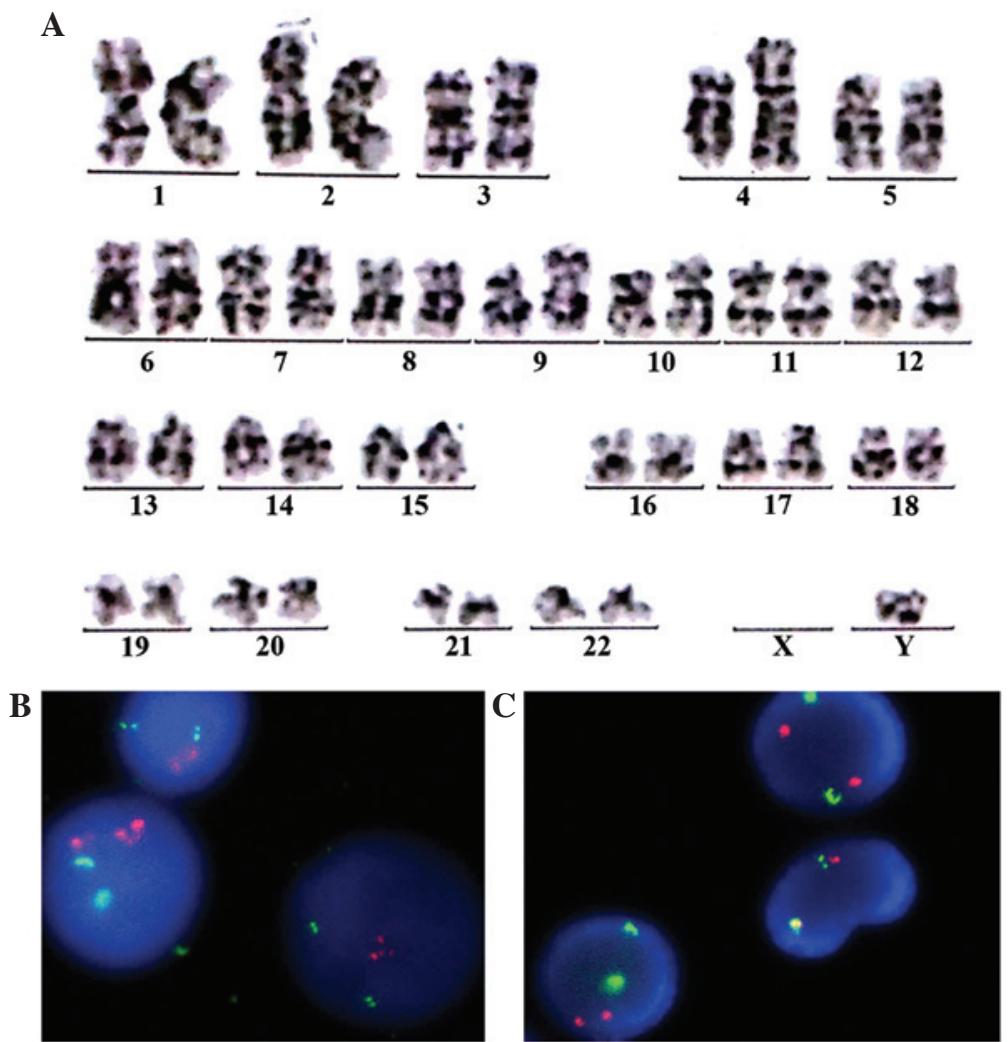

Figure 10. Result of cytogenetic analysis. (A) Karyotype 46,XY,add(4)(p16)(2)/46,XY(5) (a fragment from an unknown source that was an addition to chromosome 4 was observed in 2 out of the 7 analyzed cells. The absence of chromosome $\mathrm{X}$ was considered as a random chromosome loss). Negative fluorescence in situ hybridization for (B) BCR/ABL and (C) IGH/CCND1 genes. 

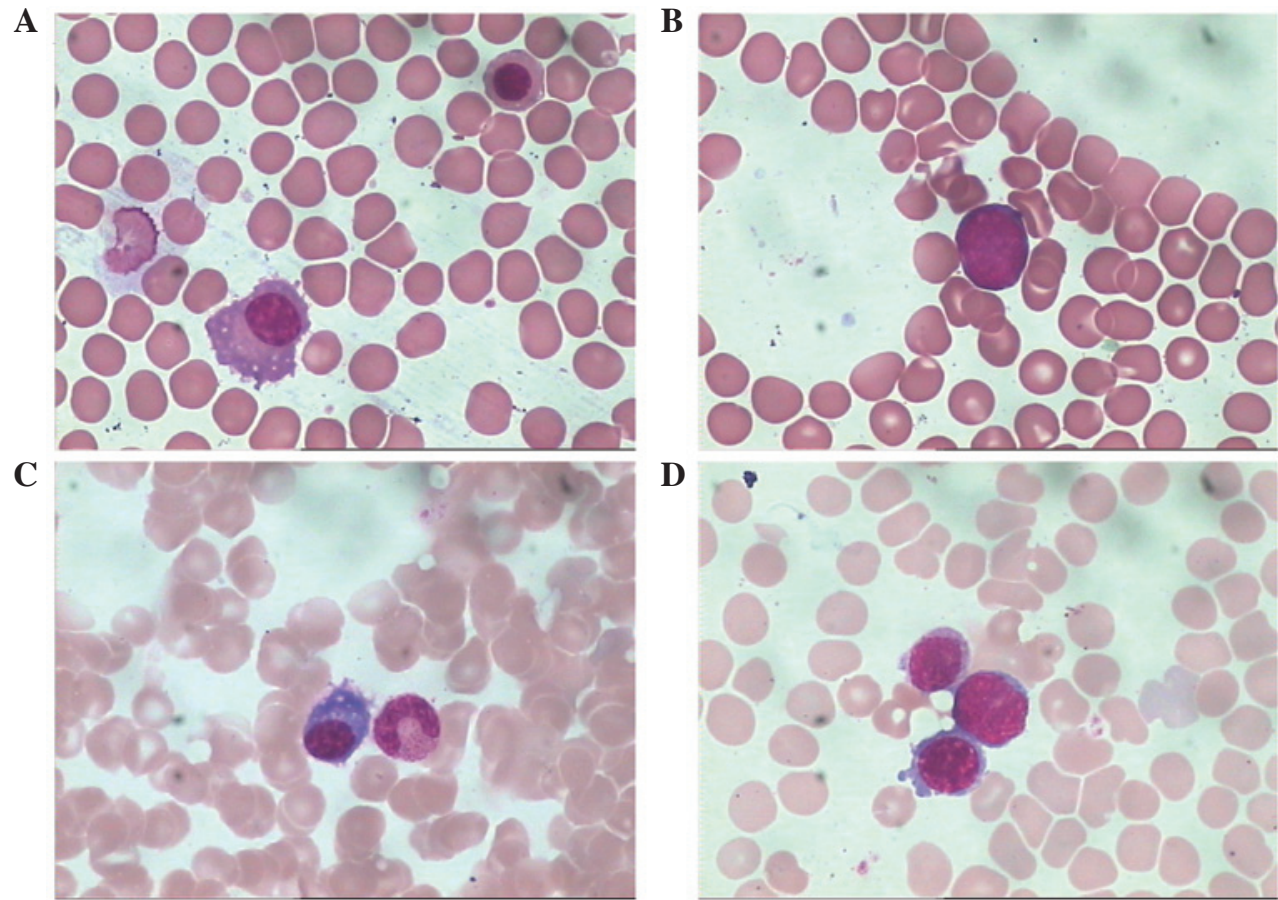

Figure 11. Cell morphology in bone marrow smears during maintenance therapy (Wright-Giemsa staining; magnification, x1,000). Images of bone marrow smears sampled on (A and B) October 13, 2011 and on (C and D) March 7, 2012. Images of the (A and C) plasma and (B and D) blast cells. The percentage and morphology were normal. The percentage of the blast cells was $<0.005 \%$. The blast cells exhibited scant agranular cytoplasm, no Auer rods, coarse to fine chromatin and indistinct nucleoli.

by an alcohol series for dehydration. Co-denaturation was conducted for $5 \mathrm{~min}$ at $75^{\circ} \mathrm{C}$, followed by overnight hybridization at $37^{\circ} \mathrm{C}$. Evaluation of the FISH signals was performed using fluorescence microscopy (Axio Imager A1; Zeiss AG, Oberkochen, Germany) under x1,000 magnification. For each test, a minimum of 200 interphase cells were evaluated for signal pattern. According to the aforementioned results, the patient was diagnosed with secondary B-cell lymphoblastic leukemia in MM.

Findings in reviewing the bone marrow smears. After secondary lymphoblastic leukemia was diagnosed, all the records of the patient were reviewed, notable among these were the bone marrow smears. The patient had six marrow sampling during maintenance therapy. All the bone marrow smears appeared in a good state, and the percentage and morphology of the plasma cells were normal. Unexpectedly, an extremely low percentage $(<0.01 \%)$ of blast cells was found in all the bone marrow smears during the maintenance therapy (Fig. 11). The blast cells had scant agranular cytoplasm, no Auer rods, coarse to fine chromatin and indistinct nucleoli. The blast cells were not noticed during maintenance therapy due to their low percentage.

The patient was then administered CHOP regimen (cyclophosphamide, $1.2 \mathrm{~g}$ on day 1; pirarubicin; $60 \mathrm{mg}$ on day 1 , vincristine, $2 \mathrm{mg}$ on day 1 ; and prednisone, $30 \mathrm{mg}$ bid on days 1-5) for 1 cycle. However, the regimen had poor efficiency, and the lymphoblasts still accounted for $48 \%$ of all nucleated cells in the patient's bone marrow smear. Subsequently, the patient discontinued the treatment and was not further followed up. The patient succumbed to disease on April 2014.

\section{Discussion}

In recent years, the application of novel agents has prolonged the survival time of MM patients, but a concerning finding has been the increase in the incidence of secondary malignancies (4-7). In the reported cases, the majority of secondary malignancies in MM were acute myelocytic leukemia (AML), myelodysplastic syndrome (MDS) and solid tumors (4-7). In a previous study, the present authors reported 3 cases of MM who developed lymphoblastic leukemia after exposure to a variety of agents (9). The present study reports the case of a patient who developed secondary lymphoblastic leukemia 38 months after the initial MM diagnosis.

It has been hypothesized that plasma cell dyscrasias exhibited a higher risk of associated AML/MDS. A number of immune system defects have been described in MM, although the clinical importance of these defects remains unclear. The more well-known of these immune defects include impaired lymphocyte function, decreased $\gamma$ globulin levels and steroid-associated lymphopenia/immunosuppression $(4,11)$. These alterations are likely to predispose MM patients to other malignancies.

Bortezomib was the main drug used in induction therapy for the present study patient. There is little literature on the secondary malignancies that are triggered by bortezomib, and available studies show that bortezomib exhibits a relatively low incidence of secondary malignancies $(12,13)$. The present patient had only a short exposure time to bortezomib ( $<2$ months).

The consolidation and maintenance therapy of the patient was somehow complicated, since after complete remission was achieved, lenalidomide, maphalan and thalidomide were 
successively administered for consolidation and maintenance therapy.

According to contemporary research, exposure to melphalan (11,13-15) and lenalidomide (15-21) may cause immunosuppression, and may be associated with a higher frequency of secondary malignancies. However, the exposure times to lenalidomide and melphalan in the present study were short (4 days and 2 months, respectively).

In the present study, the relatively long exposure time to thalidomide (31 months as maintenance therapy) was considered as an important potential risk factor. The clinical observation of patients treated with thalidomide has suggested that this agent could exert immunostimulatory and immunosuppressive activities $(22,23)$. However, thalidomide has been associated with a relatively low incidence of secondary malignancies in MM $(24,25)$.

According to the aforementioned analysis, it was difficult to determine whether any of the single agents that were used in the treatment were the cause of the secondary lymphoblastic leukemia in the present patient. It is worth noting that the treatment of the patient was somehow complex, since bortezomib, dexamethasone, lenalidomide, vincristine, pirarubicin, melphalan and thalidomide were used successively in the induction therapy, consolidation therapy and maintenance therapy. Owing to the exposure of the patient to a variety of agents, it could be inferred that multiple immune defects may exist and may play an important role in the secondary lymphoblastic leukemia that was diagnosed.

After scrutinizing the patient's bone marrow smears during the maintenance therapy, an extremely low percentage of blast cells was unexpectedly found each time. As the blast cells were at a low percentage, they were not noticed during the maintenance therapy. Therefore, flow cytometry and peroxidase staining were not performed at this time, and it was impossible to identify whether the blast cells were lymphoblasts or myeloblasts by immunophenotype. However, according to the morphology of the blast cells (scant agranular cytoplasm, no Auer rods, coarse to fine chromatin and indistinct nucleoli), we speculated that the blast cells may be lymphoblasts. It could be inferred that the patient was possibly in an early stage of lymphoblastic leukemia during the maintenance therapy of MM. If the bone marrow smears had been scrutinized carefully, and if flow cytometry had been used, an early stage of lymphoblastic leukemia could have been identified and interventional treatment could have been applied.

The present authors previously reported 3 cases of MM who developed secondary lymphoblastic leukemia. A very low percentage of similar blast cells were found in the bone marrow smears during maintenance therapy in all those 3 cases (9). It is not known whether the subsequent occurrence of secondary B-cell lymphoblastic leukemia represents a transformation of MM into a less differentiated B-cell malignancy, a biclonal neoplasm arising from an oncogenic event in a common B-cell precursor, or an independent oncogenic event due to the defect in immune dysregulation (4,6-9). However, if a low percentage of blast cells is noticed early on, flow cytometry may be introduced and provide further evidence of disease, and the patient may also have a chance for early intervention. The previous 3 cases and the present case illustrate the value of microscopic examination and flow cytometry detection in identifying secondary malignancies in MM.

In summary, the present study reports a rare case of secondary B-cell lymphoblastic leukemia that occurred 38 months after the primary diagnosis of MM, the cause of which may be associated with exposure to a variety of agents. Microscopic examination and flow cytometry detection were important in identifying the secondary malignancy in this MM case.

\section{References}

1. Junxun L, Juan L, Xiuzhen T, Juan O, Bohuang Z and Junru L: Comparing five diagnostic criteria for multiple myeloma: A retrospective study of 227 cases. Tumori 100: 207-213, 2014.

2. Smith L, McCourt O, Henrich M, Paton B, Yong K, Wardle J and Fisher A: Multiple myeloma and physical activity: A scoping review. BMJ Open 5: e009576, 2015.

3. Li J, Chen S, Hu Y and Cai J: Bortezomib-induced severe pulmonary complications in multiple myeloma: A case report and literature review. Ocol Lett 11: 2255-2260, 2016.

4. Mailankody S, Pfeiffer RM, Kristinsson SY, Korde N, Bjorkholm M, Goldin LR, Turesson I and Landgren O: Risk of acute myeloid leukemia and myelodysplastic syndromes after multiple myeloma and its precursor disease (MGUS). Blood 118: 4086-4092, 2011.

5. Pan B and Lentzsch S: The application and biology of immunomodulatory drugs (IMiDs) in cancer. Pharmacol Ther 136: 56-68, 2012.

6. Ormerod A, Fausel CA, Abonour R and Kiel PJ: Observations of second primary malignancy in patients with multiple myeloma. Clin Lymphoma Myeloma Leuk 12: 113-117, 2012.

7. Srivastava G, Rana V, Lacy MQ, Buadi FK, Hayman SR, Dispenzieri A, Gertz MA, Dingli D, Zeldenrust S, Russell S, et al: Long-term outcome with lenalidomide and dexamethasone therapy for newly diagnosed multiple myeloma. Leukemia 27: 2062-2066, 2013.

8. Gökbuget N: How I treat older patients with ALL. Blood 122: 1366-1375, 2013

9. Junxun L, Junru L, Meilan C, Chujia L, Shaoqian C, Jieyu Z, Zhuangjian Y, Fan Z, Juan O, Jing C and Juan L: Three patients with multiple myeloma developing secondary lymphoblastic leukemia: Case reports and review of the literature. Tumori: Jul 2, 2015 (Epub ahead of print). doi: 10.5301/tj.5000377.

10. Katzel JA, Hari P and Vesole DH: Multiple myeloma: Charging toward a bright future. CA Cancer J Clin 57: 301-318, 2007.

11. Schütt P, Brandhorst D, Stellberg W, Poser M, Ebeling P, Müller S, Buttkereit U, Opalka B, Lindemann M, Grosse-Wilde H, Seeber S, et al: Immune parameters in multiple myeloma patients: Influence of treatment and correlation with opportunistic infections. Leuk Lymphoma 47: 1570-1582, 2006.

12. Chanan-Khan A, Sonneveld P, Schuster MW, Stadtmauer EA, Facon T, Harousseau JL, Ben-Yehuda D, Lonial S, Goldschmidt H, Reece D, et al: Analysis of herpes zoster events among bortezomib-treated patients in the phase III APEX study. J Clin Oncol 26: 4784-4790, 2008.

13. San Miguel JF, Schlag R, Khuageva NK, Dimopoulos MA, Shpilberg O, Kropff M, Spicka I, Petrucci MT, Palumbo A, Samoilova OS, et al: Persistent overall survival benefit and no increased risk of second malignancies with bortezomib-melphalan-prednisone versus melphalan-prednisone in patients with previously untreated multiple myeloma. J Clin Oncol 31: 448-455, 2013.

14. Cuzick J, Erskine S, Edelman D and Galton DA: A comparison of the incidence of the myelodysplastic syndrome and acute myeloid leukaemia following melphalan and cyclophosphamide treatment for myelomatosis. A report to the Medical Research Council's working party on leukaemia in adults. Br J Cancer 55: 523-529, 1987.

15. Bergsagel DE, Bailey AJ, Langley GR, MacDonald RN, White DF and Miller AB: The chemotherapy on plasma-cell myeloma and the incidence of acute leukemia. N Engl J Med 301: 743-748, 1979. 
16. Dasanu CA: Immune alterations in untreated and treated multiple myeloma. J Oncol Pharm Pract 18: 257-263, 2012.

17. Tai YT, Li XF, Catley L, Coffey R, Breitkreutz I, Bae J, Song W, Podar K, Hideshima T, Chauhan D, et al: Immunomodulatory drug lenalidomide (CC-5013, IMiD3) augments anti-CD40 SGN-40-induced cytotoxicity in human multiple myeloma: Clinical implications. Cancer Res 65: 11712-11720, 2005.

18. Dasanu CA and Alexandrescu DT: A case of severe aplastic anemia secondary to treatment with lenalidomide for multiple myeloma. Eur J Haematol 82: 231-234, 2009.

19. Attal M, Lauwers-Cances V, Marit G, Caillot D, Moreau P, Facon T, Stoppa AM, Hulin C, Benboubker L, Garderet L, et al; IFM Investigators: Lenalidomide maintenance after stem-cell transplantation for multiple myeloma. N Engl J Med 366: 1782-1791, 2012.

20. McCarthy PL, Owzar K, Hofmeister CC, Hurd DD, Hassoun H, Richardson PG, Giralt S, Stadtmauer EA, Weisdorf DJ, Vij R, et al: Lenalidomide after stem-cell transplantation for multiple myeloma. N Engl J Med 366: 1770-1781, 2012.

21. Palumbo A, Hajek R, Delforge M,Kropff M,Petrucci MT, Catalano J, Gisslinger H, Wiktor-Jedrzejczak W, Zodelava M, Weisel K, et al MM-015 Investigators: Continuous lenalidomide treatment for newly diagnosed multiple myeloma. N Engl J Med 366: 1759-1769, 2012.
22. Haslett PA, Corral LG, Albert M and Kaplan G: Thalidomide costimulates primary human $\mathrm{T}$ lymphocytes, preferentially inducing proliferation, cytokine production, and cytotoxic responses in the $\mathrm{CD}^{+}$subset. J Exp Med 187: 1885-1892, 1998.

23. Verbon A, Juffermans NP, Speelman P, van Deventer SJ, ten Berge IJ, Guchelaar HJ and van der Poll T: A single oral dose of thalidomide enhances the capacity of lymphocytes to secrete gamma interferon in healthy humans. Antimicrob Agents Chemother 44: 2286-2290, 2000.

24. Usmani SZ, Sexton R, Hoering A, Heuck CJ, Nair B, Waheed S, Al Sayed Y, Chauhan N, Ahmad N, Atrash S, et al: Second malignancies in total therapy 2 and 3 for newly diagnosed multiple myeloma: Influence of thalidomide and lenalidomide during maintenance. Blood 120: 1597-1600, 2012.

25. Stewart AK, Trudel S, Bahlis NJ, White D, Sabry W, Belch A, Reiman T, Roy J, Shustik C, Kovacs MJ, et al: A randomized phase 3 trial of thalidomide and prednisone as maintenance therapy after ASCT in patients with MM with a quality-of-life assessment: The National Cancer Institute of Canada Clinicals Trials Group Myeloma 10 Trial. Blood 121: 1517-1523, 2013. 